\title{
THE FIRST RESULTS OF PROCESSING OBSERVATIONS OF SS BODIES FROM UBAI PHOTOGRAPHIC PLATE COLLECTION USING THE NEW TECHNIQUE
}

\author{
O.M. Yizhakevych ${ }^{1}$, V.M. Andruk ${ }^{1}$, Q. Yuldoshev ${ }^{2}$, L.K. Pakuliak ${ }^{1}$, M.M. Muminov ${ }^{3}$ \\ ${ }^{1}$ Main Astronomical Observatory NASU, Kyiv,Ukraine, izhak@mao.kiev.ua \\ ${ }^{2}$ Ulugh Beg Astronomical Institute UzAS,Tashkent, Uzbekistan,qudratillo@astrin.uz \\ ${ }^{3}$ Andijan State University, Uzbekistan, Andijan, muminov1951@gmail.com
}

ABSTRACT. The paper presents the first results of processing of photographic observations of SS bodies based on materials from the collection of plates of the Ulugh Beg Astronomical Institute of the Academy of Sciences of the Republic of Uzbekistan (UBAI). The glass archive of the UBAI has about 15 thousand photographic plates with images of various space objects. In this paper, we discuss the first results of processing of major planets' photographic observations in Uzbekistan using the new technique. The observations were carried out on two astrographs - Tashkent normal astrograph (TNA, $\mathrm{D} / \mathrm{F}=330 / 3438$ ) and Kitab Double Zeiss astrograph (DAZ, D/F=400/3000) in 1895-2004. Currently, in AI the work runs to streamline the archive and create a database of observations in a single xls-format. The number of photographic plates with images of major planets obtained on two telescopes is more than 300 negatives. In 2017, together with MAO NAS of Ukraine, the processing of these photographic observations using the new methodology was started. By this time, in the framework of the UkrVO project (Vavilova, 2012a; Vavilova, 2012b; Vavilova, 2014; Vavilova, 2016; Vavilova, 2017) the method of reducing digitized plates was developed for the FON program. The plate digitization is performed on the flat-bed 10000XL in 1200dpi mode. Positions and photometric estimations in B-color of all registered on the plate objects are obtained using the software proposed and developed in MAO NAS of Ukraine running in the LINUX/MIDAS/ROMAPHOT complex.. The search for major planets and their satellites among the whole set of plate objects and the comparison of their observed and predicted positions is carried out online with the help of internet-service (http://nfm1.sai.msu.ru/neb/nss/nssephmr.htm). Up to date, the small part of the plate collection was treated, namely 23 negatives with images of Uranus, Neptune, Pluto and Saturn's satellites, obtained on the Kitab DAZ astrograph. 16 negatives with 19 exposures gave the best results with $(\mathrm{O}-\mathrm{C})$ in the range $\pm 1 . " 5$. In total, we obtained 47 astrometric positions of the objects of interest in the wide interval of brightness's $\left(8^{\mathrm{m}} \pm 16^{\mathrm{m}}\right)$. The internal positional accuracy of the catalog in the Tycho-2 reference system is $\sigma_{\alpha \delta}=0.08^{\prime \prime}-0.13^{\prime \prime}$, the photometric error of B-magnitudes is estimated as $\sigma_{\mathrm{B}}=0.2^{\mathrm{m}}$.

Keywords: digital plate processing - astrometry of Major planets - satellites - catalogues.

АБСТРАКТ. Архів склотеки Астрономічного інституту Академії Наук Республіки Узбекістан (UBAI) нараховує майже 15 тисяч фотопластинок із зображеннями різних об'єктів Космосу. В роботі йдеться про перші результати опрацювання спостережень Великих планет та їхніх супутників, зображення яких було отримано за допомогою двох астрографів - Ташкентського нормального астрографа (TNA) та Кітабського Подвійного астрографа Цейса (DAZ) в Узбекистані у подовж 1895-2004 років. На даний час в AI виконуються роботи 3 упорядкування архіву платівок та створення Банка даних фотографічних спостережень у єдиному xlsформаті. Кількість фотопластинок із зображеннями Великих Планет становить понад 300 негативів. У 2017 році в АІ спільно з ГАО НАНУ розпочато опрацювання цих спостережень за новою методою. На той час, у рамках проекту УкрВО (Vavilova, 2012a; Vavilova, 2012b; Vavilova, 2014; Vavilova, 2016; Vavilova, 2017) був розроблений та запропонований метод редукції оцифрованих пластинок для програми ФОН. Оцифрування негативів здійснюється за допомогою планшетного сканера Epson Expression 10000XL. в режимі 1200dpi. Подальша редукція оцифрованих платівок відбувається завдяки комплексу програм, спеціально розроблених в ГАО в операційному середовищі LINUX/MIDAS/ROMAFOT. За допомогою інтернет-сайта (http://nfm1.sai.msu.ru/neb/nss/nssephmr.htm) в режимі online відшукуємо зображення Планет серед усіх об'єктів платівки та визначаємо розбіжності $(\mathrm{O}-\mathrm{C})^{\prime \prime}$ між обчисленими та їх теоретичними положеннями. На даний момент опрацьовано невелику частку спостережного матеріалу (23 негатива) із зображеннями Урана. Нептуна, Плутона та супутників Сатурна, що були отримані на астрографі DAZ в Кітабі. Успішними виявилися 16 фотопластинок (19 знімків), для яких значення (О-C) не перевищує $\pm 1.5^{\prime \prime}$. Загалом, ми отримали 47 астрометричних положень Планет в 
широкому діапазоні їх яскравості $\left(8^{\mathrm{m}} \div 16^{\mathrm{m}}\right)$. Внутрішня точність редукції RMS по обох координатах знаходиться в межах $\sigma_{\alpha \delta}=0.08^{\prime \prime} \div 0.13^{\prime \prime}$, а похибка визначення зоряної величини становить $\sigma_{\mathrm{B}}=0.2^{\mathrm{m}}$.

Ключові слова: Опрацювання оцифрованих пластинок - астрометрія - великі планети - супутники - каталоги.

\section{Introduction}

The glass archive of UBAI possesses a vast collection of photographic observations having been obtained since 1895. The total number of direct plates with selected sky areas is 15 thousand. Observations on the Tashkent normal astrograph (TNA) were successfully performed until 1986. Later, the main observational activity was moved to the Kitab observational site. Here, in 1975 the Zeiss Double Wide-Angle astrograph DAZ (Yuldoshev, 2016; Yuldoshev, 2017a; Yuldoshev, 2017b) was installed for the observations on the FON project (Photographic survey of the northern sky) (Kolchinskiy, 1977; Pakuliak, 2016; Andruk, 2017b). Below, Table 1 gives the parameters of both telescopes. The archive of photographic plates got on DAZ in 2015 was moved from Kitab to Tashkent. Later, the works run to create the data bank of all observations in the single xls-format. In 2017, the photographic plate processing has been initiated together with MAO NAS of Ukraine. The archive of photographic plates got on DAZ in 2015 was moved from Kitab to Tashkent. Later, the works run to create the data bank of all observations in the single xls-format.

Table1. Parameters of TNA and DAZ telescopes

\begin{tabular}{|c|c|c|}
\hline Parameter & $\begin{array}{c}\text { Ташкент, } \\
\text { TNA }\end{array}$ & Kitab, DAZ \\
\hline ID & TAS033 & $\begin{array}{c}\text { TAS040A } \\
\text { TAS040B }\end{array}$ \\
\hline $\begin{array}{c}\text { Marsden's } \\
\text { Code }\end{array}$ & 192 & 186 \\
\hline Longitude & $69^{\circ} 17 .^{\prime} 0$ & $66^{\circ} 53 .^{\prime} 0$ \\
\hline Latitude & $41^{\circ} 19 .^{\prime} 5$ & $39^{\circ} 08 .^{\prime} 0$ \\
\hline Altitude & $482 \mathrm{~m}$ & $690 \mathrm{~m}$ \\
\hline Aperture & $0.33 \mathrm{~m}$ & $0.40 \mathrm{~m}$ \\
\hline Focal length & $3.43 \mathrm{~m}$ & $3 \mathrm{~m}$ \\
\hline Scale & $60^{\prime \prime} / \mathrm{mm}$ & $69^{\prime \prime} / \mathrm{mm}$ \\
\hline Field & $2^{\circ} \div 2.5^{\circ}$ & $5.5^{\circ} \div 6.0^{\circ}$ \\
\hline $\begin{array}{c}\text { Glass plate } \\
\text { size (max) }\end{array}$ & $16 \times 16 \mathrm{~cm}$ & $30 \times 30 \mathrm{~cm}$ \\
\hline
\end{tabular}

In 2017, the photographic plate processing has been initiated together with MAO NAS of Ukraine. The objects of interest were plates with images of major planets obtained in Uzbekistan on two telescopes. The renovation of the Institute building has temporarily complicated the access to the observational material. So, only 23 plates with Kitab DAZ observations were available and had been processed up to date.

\section{The procedure of major planets' observation} processing

The plate digitization was made using flat-bed commercial scanner Epson Expression 10000XL in grey color range 16-bits with a spatial resolution 1200 dpi (Protsyuk, 2014; Eglitis, 2017). The flat-bed scanner accompanied with the proper software allows digitizing and processing of plates containing objects of any brightnesses. The astrometric reduction of digitized data was made using the software proposed and developed in MAO NAS of Ukraine for the FON project (Relke, 2015; Andruk, 2015; Andruk, 2016a; Andruk2016b; Andruk, 2017a). The total package runs in LINUX/MIDAS/ROMAFOT operational environment. The initial scripts of the package were modified to meet the requirements of SS bodies' specific observations. In our case, the internal positional accuracy of the reduction lies in the range $\sigma_{\alpha \delta}=0.10^{\prime \prime}$, the accuracy of photometric estimations is better then $\sigma_{\mathrm{B}}=0.2^{\mathrm{m}}$. Table 2 contains the results of processing 16 plates with Neptune, Uranus and its satellite Oberon, Pluto, and five Saturn's moons. The catalog of observed topocentric positions and the results of their comparison with theoretic data is given in Table 3. The observations which have the discrepancies with the theory more than \pm 2.5 arcsec were not included to the catalog. The plates required the more accurate moments of observations were excluded as well.

\section{Conclusions}

The first 47 topocentric positions of major planets and their satellites were obtained from DAZ observations. The processing of photographic observational data was made using algorithms of digital data reduction and the software developed in MAO NAS of Ukraine. Earlier, the same technique was tested and proved for the search for asteroids and satellites of major planets (Protsyuk, 2017; Shatokhina, 2016; Shatokhina, 2017; Yizhakevych, 2014; Yizhakevych, 2015; Yizhakevych, 2016; Yizhakevych, 2017a; Yizhakevych, 2017b). The comparison of observed positions of objects with those of online ephemeris data of IMCCE demonstrates the good agreement of the theory and observations.

In the future, we plan to go on with the processing of photographic plates from UBAI glass collection containing the images of Solar system bodies obtained in Uzbekistan on two astrographs.

\section{References}

Andruk V.M., Pakuliak L.K., Golovnia V.V. et al.: 2015, Odessa Astron. Publ., 28, 192.

Andruk V.M., Golovnia V.V., Ivanov G.A. et al.: 2016, Kinem. Phys. Cel. Bodies, 32, N1, 38.

Andruk V.M., Pakuliak L.K., Golovnia V.V. et al.: 2016, Kinem. Phys. Cel. Bodies, 32, N5, 260.

Andruk V.M., Pakuliak L.K., Golovnia V.V. et al.: 2017, Scince and Innovation, 13a, 17.

Andruk V., Yuldoshev Q., Eglitis I. et al.: 2017, Odessa Astron. Publ., 30, 159.

Eglitis I., Andruk V.: 2017, Open Astronomy, 26, N1, 7.

Kolchinsky I.G., Onegina A.B.: 1977, Astrometry and Astrophysics, 33, 11.

Pakuliak L.K., Andruk V.M., Golovnia V.V. et al.: 2016, Odessa Astron. Publ., 29, 132.

Protsyuk Yu.I., Andruk V.N., Muminov M.M. et al.: 2014, Odessa Astron. Publ., 27, 61. 
Table 2. The statistical data of the reduction of SS bodies observations made in Kitab (DAZ):

1 -object name; 2-years of observations; 3 - number of plates /number of observational nights;

4 - number of positions, 5 - photographic stellar magnitude; 6,8,10-standard deviation; 7,9-(O-C) on right ascension and declination; 11, 12,13-RMS-internal error of the reduction;

14 - number of reference stars from Tycho-2.

\begin{tabular}{|c|c|c|c|c|c|c|c|c|c|c|c|c|c|}
\hline \multirow[b]{2}{*}{ Obj } & \multirow[b]{2}{*}{ Range } & \multirow{2}{*}{$\begin{array}{c}\text { N pl. } \\
/ \mathbf{N} \\
\text { nights }\end{array}$} & \multirow{2}{*}{$\begin{array}{c}\mathrm{N} \\
\text { pos. }\end{array}$} & \multirow[b]{2}{*}{ Bph } & \multirow{2}{*}{$\begin{array}{l}\text { Sd } \\
\text { Bph }\end{array}$} & \multirow{2}{*}{$\begin{array}{l}\text { O-C } \\
\text { R.A. }\end{array}$} & \multirow{2}{*}{$\begin{array}{r}\text { Sd } \\
\text { R.A. }\end{array}$} & \multirow{2}{*}{$\begin{array}{c}\text { O-C } \\
\text { Del. }\end{array}$} & \multirow{2}{*}{$\begin{array}{l}\text { Sd } \\
\text { Del }\end{array}$} & \multicolumn{3}{|c|}{ RMS } & \multirow[b]{2}{*}{$\mathbf{n}$} \\
\hline & & & & & & & & & & Bph & R.A & Del & \\
\hline 1 & 2 & 3 & 4 & 5 & 6 & 7 & 8 & 9 & 10 & 11 & 12 & 13 & 14 \\
\hline \multicolumn{14}{|c|}{ SATURN'S MOONS } \\
\hline S5 & $\begin{array}{l}1983, \\
1990\end{array}$ & $4 / 4$ & 8 & 10.3 & .44 & -0.53 & .77 & +0.36 & .56 & .29 & .08 & .09 & 1277 \\
\hline S6 & $\begin{array}{l}1983, \\
1986, \\
1990\end{array}$ & $6 / 6$ & 10 & 9.9 & .19 & +0.13 & .59 & -0.04 & .36 & .28 & .09 & .09 & 1194 \\
\hline S7 & 1990 & $3 / 3$ & 3 & 15.0 & .20 & -0.00 & .35 & -0.09 & .14 & .28 & .07 & .08 & 1725 \\
\hline S8 & 1990 & $4 / 4$ & 6 & 12.8 & .20 & +0.05 & .22 & -0.19 & .31 & .29 & 09 & .10 & 1676 \\
\hline S9 & 1990 & $1 / 1$ & 1 & 16.6 & & +3.96 & & +1.04 & & .28 & .06 & .07 & 1675 \\
\hline$\Sigma$ & $\begin{array}{c}1983- \\
1990\end{array}$ & $6 / 6$ & 27 & & & & & & & & & & \\
\hline \multicolumn{14}{|c|}{ URANUS } \\
\hline $\begin{array}{l}\text { Ura- } \\
\text { nus }\end{array}$ & $\begin{array}{c}1983, \\
1985\end{array}$ & $3 / 3$ & 6 & 7.5 & 1.34 & -0.13 & .57 & -0.35 & .34 & .33 & .13 & .14 & 630 \\
\hline U4 & $\begin{array}{l}1979, \\
1983 \\
1985\end{array}$ & $4 / 4$ & 4 & 13.9 & 0.72 & +0.13 & .94 & +0.44 & .82 & .30 & .08 & .08 & 729 \\
\hline$\Sigma$ & $\begin{array}{c}1979- \\
1985\end{array}$ & $7 / 7$ & 10 & & & & & & & & & & \\
\hline \multicolumn{14}{|c|}{ NEPTUNE } \\
\hline $\begin{array}{l}\text { Nep- } \\
\text { tune }\end{array}$ & $\begin{array}{c}1983, \\
1984\end{array}$ & $4 / 4$ & 9 & 8.4 & 0.48 & +0.05 & .64 & 0.11 & .45 & .33 & .13 & .14 & 2245 \\
\hline \multicolumn{14}{|c|}{ PLUTO } \\
\hline $\begin{array}{l}\text { Plu } \\
\text {-to }\end{array}$ & 1990 & $2 / 2$ & 3 & 13.7 & 0.15 & -0.19 & .05 & -0.25 & .19 & .29 & .06 & .07 & 527 \\
\hline
\end{tabular}

Table 3. The catalog of topocentric positions of major planets and their satellites from observations on DAZ astrograph in Kitab: 1 -Date $+U T$ in fractions of day; 2 - Right Ascension (h,m,sec); 3 -Declination (degrees, min.,arcsec); 4 - exposure (min.); 5 -photographic magnitude; 6,7-(O-C) on Right Ascension and Declination (arcsec); 8 - number of plate.

\begin{tabular}{|c|c|c|c|c|c|c|c|c|c|c|c|c|c|}
\hline \multicolumn{3}{|c|}{ Date, UT } & \multicolumn{3}{|c|}{ RA } & \multicolumn{3}{|c|}{ DEC } & Exp. & $\mathbf{B}_{\mathrm{ph}}$ & $(\mathbf{O}-\mathrm{C})_{\alpha}$ & $(\mathrm{O}-\mathrm{C})_{\delta}$ & NPI. \\
\hline \multicolumn{3}{|c|}{1} & \multicolumn{3}{|c|}{2} & \multicolumn{3}{|c|}{3} & 4 & 5 & 6 & 7 & 8 \\
\hline$Y$ & $M$ & Day & $h$ & $m$ & $s$ & $\circ$ & ' & $"$ & $\min$ & mag & $"$ & $"$ & \\
\hline \multicolumn{14}{|c|}{ NEPTUNE } \\
\hline 1983 & 09 & 11.656048 & 17 & 45 & 44.237 & -22 & 10 & 49.307 & 15 & 8.7 & 1.06 & 0.10 & 17 \\
\hline 1984 & 06 & 21.762796 & 18 & 01 & 4.906 & -22 & 13 & 29.341 & 2 & 8.6 & 0.22 & 0.64 & 1645 \\
\hline 1984 & 06 & 21.769491 & 18 & 01 & 4.904 & -22 & 13 & 29.758 & 15 & 7.6 & 0.91 & 0.23 & 1645 \\
\hline 1984 & 06 & 21.775954 & 18 & 01 & 4.822 & -22 & 13 & 30.408 & 2 & 8.6 & 0.37 & -0.42 & 1645 \\
\hline 1984 & 06 & 24.795219 & 18 & 00 & 43.611 & -22 & 13 & 32.322 & 2 & 8.8 & -0.62 & -0.10 & 1753 \\
\hline 1984 & 06 & 24.801625 & 18 & 00 & 43.592 & -22 & 13 & 32.551 & 15 & 7.6 & -0.22 & -0.33 & 1753 \\
\hline 1984 & 06 & 24.814891 & 18 & 00 & 43.552 & -22 & 13 & 31.947 & 2 & 8.7 & 0.59 & 0.29 & 1753 \\
\hline 1984 & 06 & 27.781188 & 18 & 00 & 22.902 & -22 & 13 & 34.920 & 36 & 8.8 & 1.20 & -0.28 & 1718 \\
\hline 1984 & 06 & 27.800493 & 18 & 00 & 22.762 & -22 & 13 & 33.755 & 5 & 8.5 & 1.15 & 0.90 & 1718 \\
\hline \multicolumn{14}{|c|}{ PLUTO } \\
\hline 1990 & 06 & 13.778986 & 15 & 10 & 36.276 & -01 & 17 & 4.240 & 30 & 13.9 & -0.23 & -0.47 & 1822 \\
\hline 1990 & 07 & 17.722097 & 15 & 08 & 38.607 & -01 & 25 & 31.984 & 21 & 13.6 & -0.22 & -0.14 & 1850 \\
\hline 1990 & 07 & 17.725559 & 15 & 08 & 38.613 & -01 & 25 & 32.081 & 21 & 13.7 & -0.13 & -0.14 & 1850 \\
\hline
\end{tabular}




\begin{tabular}{|c|c|c|c|c|c|c|c|c|c|c|c|c|c|}
\hline \multicolumn{14}{|c|}{ U4 OBERON } \\
\hline 1979 & 07 & 14.693111 & 14 & 59 & 34.990 & -16 & 41 & 24.500 & 15 & 13.5 & -0.60 & 1.44 & 470 \\
\hline 1983 & 07 & 2.686363 & 16 & 16 & 37.989 & -21 & 12 & 50.224 & 15 & 13.8 & -0.52 & -0.56 & 1529 \\
\hline 1985 & 09 & 5.651112 & 16 & 51 & 39.235 & -22 & 32 & 55.360 & 15 & 15.0 & 0.12 & 0.38 & 1376 \\
\hline \multicolumn{14}{|c|}{ URANUS } \\
\hline 1983 & 04 & 14.934433 & 16 & 28 & 57.093 & -21 & 41 & 18.023 & 17 & 5.6 & 0.23 & -0.60 & 1619 \\
\hline 1983 & 04 & 14.941162 & 16 & 28 & 56.967 & -21 & 41 & 17.559 & 3 & 6.6 & -0.98 & -0.23 & 1619 \\
\hline 1983 & 07 & 2.686366 & 16 & 16 & 36.535 & -21 & 13 & 28.697 & 15 & 9.0 & 0.40 & -0.92 & 1529 \\
\hline 1985 & 09 & 5.641802 & 16 & 51 & 36.362 & -22 & 33 & 9.358 & 2 & 7.5 & 0.43 & -0.13 & 1376 \\
\hline 1985 & 09 & 5.644533 & 16 & 51 & 36.313 & -22 & 33 & 9.422 & 2 & 7.5 & -0.42 & -0.18 & 1376 \\
\hline 1985 & 09 & 5.648381 & 16 & 51 & 36.323 & -22 & 33 & 9.324 & 15 & 9.0 & -0.44 & -0.06 & 1376 \\
\hline \multicolumn{14}{|c|}{ S5 RHEA } \\
\hline 1983 & 04 & 13.862186 & 14 & 03 & 3.460 & -09 & 34 & 28.083 & 3 & 10.3 & -0.20 & 0.14 & 1433 \\
\hline 1983 & 04 & 13.864896 & 14 & 03 & 3.422 & -09 & 34 & 27.878 & 3 & 10.3 & -0.27 & 0.15 & 1433 \\
\hline 1983 & 04 & 13.867666 & 14 & 03 & 3.376 & -09 & 34 & 27.794 & 3 & 10.3 & -0.44 & 0.04 & 1433 \\
\hline 1990 & 07 & 14.744142 & 19 & 35 & 42.985 & -21 & 32 & 58.089 & 15 & 10.3 & -0.24 & 0.31 & 1842 \\
\hline 1990 & 07 & 16.766385 & 19 & 34 & 53.635 & -21 & 34 & 23.908 & 15 & 10.7 & 0.40 & -0.24 & 1847 \\
\hline 1990 & 07 & 19.774135 & 19 & 34 & 6.618 & -21 & 37 & 16.724 & 1 & 10.5 & -0.75 & 0.22 & 1862 \\
\hline 1990 & 07 & 19.780367 & 19 & 34 & 6.362 & -21 & 37 & 15.775 & 15 & 9.3 & -2.26 & 1.60 & 1862 \\
\hline 1990 & 07 & 19.786600 & 19 & 34 & 6.326 & -21 & 37 & 17.137 & 1 & 10.7 & -0.46 & 0.68 & 1862 \\
\hline \multicolumn{14}{|c|}{ S6 TITAN } \\
\hline 1983 & 04 & 13.862186 & 14 & 02 & 47.294 & -09 & 34 & 22.163 & 3 & 9.9 & 0.07 & -0.12 & 1433 \\
\hline 1983 & 04 & 13.864896 & 14 & 02 & 47.259 & -09 & 34 & 21.608 & 3 & 9.9 & 0.16 & 0.13 & 1433 \\
\hline 1983 & 04 & 13.867666 & 14 & 02 & 47.177 & -09 & 34 & 21.522 & 3 & 9.9 & -0.44 & -0.09 & 1433 \\
\hline 1986 & 06 & 29.724425 & 16 & 11 & 20.692 & -19 & 05 & 53.813 & 1 & 9.7 & 1.58 & -0.10 & 1425 \\
\hline 1990 & 07 & 14.744142 & 19 & 35 & 24.083 & -21 & 32 & 47.862 & 15 & 9.8 & 0.07 & -0.09 & 1842 \\
\hline 1990 & 07 & 15.732411 & 19 & 35 & 5.256 & -21 & 33 & 6.876 & 15 & 9.8 & -0.15 & 0.18 & 1844 \\
\hline 1990 & 07 & 16.766385 & 19 & 34 & 47.665 & -21 & 33 & 31.633 & 15 & 9.8 & 0.16 & -0.17 & 1847 \\
\hline 1990 & 07 & 19.774135 & 19 & 34 & 5.029 & -21 & 35 & 39.340 & 1 & 9.8 & -0.13 & 0.74 & 1862 \\
\hline 1990 & 07 & 19.780367 & 19 & 34 & 4.986 & -21 & 35 & 41.142 & 15 & 9.9 & 0.51 & -0.68 & 1862 \\
\hline 1990 & 07 & 19.786600 & 19 & 34 & 4.830 & -21 & 35 & 40.987 & 1 & 10.4 & -0.54 & -0.15 & 1862 \\
\hline \multicolumn{14}{|c|}{ S7 HYPERION } \\
\hline 1990 & 07 & 14.744142 & 19 & 35 & 24.110 & -21 & 31 & 48.484 & 15 & 15.2 & 0.17 & -0.22 & 1842 \\
\hline 1990 & 07 & 15.732411 & 19 & 35 & 8.372 & -21 & 32 & 18.102 & 15 & 15.0 & -0.41 & 0.06 & 1844 \\
\hline 1990 & 07 & 19.780367 & 19 & 34 & 10.289 & -21 & 35 & 37.961 & 15 & 14.8 & 0.23 & -0.09 & 1862 \\
\hline \multicolumn{14}{|c|}{ S8 IAPETUS } \\
\hline 1990 & 07 & 14.744142 & 19 & 36 & 13.530 & -21 & 33 & 20.375 & 15 & 12.7 & -0.02 & -0.18 & 1842 \\
\hline 1990 & 07 & 15.732411 & 19 & 35 & 55.746 & -21 & 34 & 14.853 & 15 & 12.8 & 0.36 & 0.17 & 1844 \\
\hline 1990 & 07 & 16.766385 & 19 & 35 & 36.792 & -21 & 35 & 12.787 & 15 & 12.7 & -0.18 & -0.76 & 1847 \\
\hline 1990 & 07 & 19.774135 & 19 & 34 & 40.299 & -21 & 37 & 54.795 & 1 & 12.7 & -0.04 & -0.07 & 1862 \\
\hline 1990 & 07 & 19.780367 & 19 & 34 & 40.199 & -21 & 37 & 55.180 & 15 & 12.9 & 0.28 & -0.12 & 1862 \\
\hline 1990 & 07 & 19.786600 & 19 & 34 & 40.054 & -21 & 37 & 55.598 & 1 & 13.2 & -0.07 & -0.21 & 1862 \\
\hline \multicolumn{14}{|c|}{ S9 Phoebe } \\
\hline 1983 & 07 & 11.689238 & 16 & 15 & 30.593 & -21 & 10 & 49.381 & 15 & 9.5 & 3.63 & 1.86 & 1614 \\
\hline
\end{tabular}

Protsyuk Yu.I., Kovylianska O.E., Protsyuk S.V. et al.: 2017, Scince and Innovation, 13a, 89.

Relke E., Protsyuk Yu.I., Andruk V.M.: 2015, Odessa Astron. Publ., 28, 211.

Shatokhina S.V., Kazantseva L.V., Yizhakevych O.M. et al.: 2017, Odessa Astron. Publ., 30,198.

Shatokhina S.V., Kazantseva L.V., Yizhakevych O.M. et al.: 2018, Kinem. Phys. Cel. Bodies, 34, N5, 70.

Vavilova I.B., Pakulyak L.K., Shlyapnikov A.A. et al.:2012, Kinem. Phys. Cel. Bodies, 28, N2, 85.

Vavilova I.B., Pakuliak L.K., Protsyuk Yu.I. et al.:2012, Baltic Ast., 21, N3, 356.

Vavilova, I.B., Golovnya, V.V., Andruk, V.M. et al.: 2014., Odessa Astron. Publ., 27, 132.

Vavilova I.B.: 2016, Odessa Astron. Publ., 29, 109.

Vavilova I.B., Yatskiv Ya.S., Pakuliak L.K. et al.: 2017, IAUS, 325, 361 .
Yizhakevych O.M., Andruk V.M., Pakuliak L.K. et al.: 2014, Odessa Astron Publ., 27, 67.

Yizhakevych O.M., Andruk V.M., Pakuliak L.K. 2015, Odessa Astron Publ., 28, 213.

Yizhakevych O.M., Andruk V.M., Pakuliak L.K. 2016, Odessa Astron Publ., 29, 155.

Yizhakevych O.M., Andruk V.M., Pakuliak L.K. 2017, Odessa Astron Publ., 30, 201.

Yizhakevych O.M., Andruk V.M., Pakuliak L.K. 2017, Kinem. Phys. Cel. Bodies, 33, N3, 142.

Yuldoshev Q.X., Muminov M.M., Ehgamberdiev Sh.A. et. al.: 2016, Odessa Astron Publ., 29, 160.

Yuldoshev Q.X., Muminov M.M., Ehgamberdiev Sh.A. et. al.: 2017, Odessa Astron Publ., 30, 205.

Yuldoshev Q.X., Ehgamberdiev Sh.A., Muminov M.M. et al.: 2017, Kinem. Phys. Cel. Bodies, 33, N5, 250. 